\title{
ARTICLE OPEN SARS-CoV-2 nsp12 attenuates type I interferon production by inhibiting IRF3 nuclear translocation
}

\author{
Wenjing Wang ${ }^{1,2,3}$, Zhuo Zhou ${ }^{4}$, Xia Xiao ${ }^{1,2,3}$, Zhongqin Tian ${ }^{1,2,3}$, Xiaojing Dong ${ }^{1,2,3}$, Conghui Wang ${ }^{1,2,3}$, Li Li ${ }^{1}$, Lili Ren ${ }^{1,2,3}$, \\ Xiaobo Lei ${ }^{1,2,3}$, Zichun Xiang ${ }^{1,2,3}$ and Jianwei Wang $\mathbb{i D}^{1,2,3}$
}

SARS-CoV-2 is the pathogenic agent of COVID-19, which has evolved into a global pandemic. Compared with some other respiratory RNA viruses, SARS-CoV-2 is a poor inducer of type I interferon (IFN). Here, we report that SARS-CoV-2 nsp12, the viral RNA-dependent RNA polymerase (RdRp), suppresses host antiviral responses. SARS-CoV-2 nsp12 attenuated Sendai virus (SeV)- or poly(I:C)-induced IFN- $\beta$ promoter activation in a dose-dependent manner. It also inhibited IFN promoter activation triggered by RIG-I, MDA5, MAVS, and IRF3 overexpression. Nsp12 did not impair IRF3 phosphorylation but suppressed the nuclear translocation of IRF3. Mutational analyses suggested that this suppression was not dependent on the polymerase activity of nsp12. Given these findings, our study reveals that SARS-CoV-2 RdRp can antagonize host antiviral innate immunity and thus provides insights into viral pathogenesis.

Keywords: SARS-CoV-2; COVID-19; Nsp12; Antiviral immunity

Cellular \& Molecular Immunology (2021) 18:945-953; https://doi.org/10.1038/s41423-020-00619-y

\section{INTRODUCTION}

Coronavirus disease 2019 (COVID-19) has evolved into a global pandemic. According to the WHO Coronavirus Disease dashboard, there were $63,360,234$ confirmed cases of COVID-19, including 1,475,825 deaths, as of 2 December 2020 (https://covid19.who.int/). Severe acute respiratory syndrome coronavirus 2 (SARS-CoV-2) has been identified as the pathogenic agent of COVID-19. ${ }^{1-3}$ The International Committee on Taxonomy of Viruses has classified SARS-CoV-2 into the Coronaviridae family, the Orthocoronavirinae subfamily, the Betacoronaviruses genus, and the Sarbecovirus subgenus. ${ }^{4}$

SARS-CoV-2 is a positive-stranded RNA virus with a genome of $\sim 29.7 \mathrm{~kb}^{1}$ The replicative cycle of the coronavirus initiates with the translation of ORF1a and ORF1b after entry and genome release ${ }^{5}$. Two large replicase polyproteins (pp1a and pp1ab) are first synthesized. They are then cleaved into 16 nonstructural proteins (nsps), including nsp12, a viral RNA-dependent RNA polymerase (RdRp), by papain-like protease (nsp3) and 3C-like protease (nsp5). ${ }^{5}$ Viral nsps leverage the host cell membrane structure to assemble into replication and transcription complexes that engage in minus-strand RNA synthesis., ${ }^{5,6}$ Subgenomic RNAs are then synthesized, and the structural and accessory proteins are expressed.

Nsp12 consists of 932 amino acids. The structure of nsp12 contains a nidovirus RdRp-associated nucleotidyltransferase (NiRAN) domain and a right-hand RdRp domain. ${ }^{7}$ The NiRAN domain and RdRp domain are connected by an interface domain. ${ }^{7}$ The RdRp domain contains catalytic residues (amino acids 759-761 [SDD]). ${ }^{7}$ Of note, SARS-CoV-2 RdRp has a GDD module (amino acids 823-825), whose residues function as catalytic residues in other viral RdRps, such as HCV NS5b and poliovirus $3 D^{\text {pol }} 8,9$ In addition to RNA synthesis activity, RdRps of enteroviruses, HCV, tick-borne encephalitis virus, and Langat virus regulate the host innate immune response through inhibition or activation of the expression of IFN- $\beta$ or other cytokines. ${ }^{10-13}$ In a screen for SARS-CoV-2 genes involved in modulating the host antiviral response, ${ }^{14}$ we identified that nsp12 could inhibit SeVinduced IFN- $\beta$ promoter activation, but the underlying mechanism is not clear.

The innate immune system is critical for the initial detection and restriction of virus infections. After a cell is infected by a pathogen, pattern recognition receptors, such as RIG-I-like receptors (RLRs) and Toll-like receptors, recognize pathogen-associated molecular patterns (PAMPs) immediately to activate the innate immune system. ${ }^{15-18}$ Retinoic acid-inducible gene I (RIG-I) and melanoma differentiation gene 5 (MDA5) are two cytoplasmic RLRs that recruit the adaptor protein mitochondrial antiviral signaling protein (MAVS) after PAMP recognition. ${ }^{19-22}$ MAVS then initiates signaling pathways involving multiple kinases, which lead to the phosphorylation of interferon regulatory factor $3 / 7$ (IRF3/7). ${ }^{23}$ After phosphorylation and dimerization, IRF3/7 translocates to the nucleus to promote the production of IFN- $\alpha / \beta .^{24,25}$

\footnotetext{
${ }^{1} \mathrm{NHC}$ Key Laboratory of System Biology of Pathogens, Institute of Pathogen Biology, Chinese Academy of Medical Sciences \& Peking Union Medical College, Beijing 100730, PR China; ${ }^{2}$ Key Laboratory of Respiratory Disease Pathogenomics, Chinese Academy of Medical Sciences and Peking Union Medical College, Beijing 100730 , PR China; ${ }^{3}$ Christophe Merieux Laboratory, Institute of Pathogen Biology, Chinese Academy of Medical Sciences \& Peking Union Medical College, Beijing 100730, PR China and ${ }^{4}$ Biomedical Pioneering

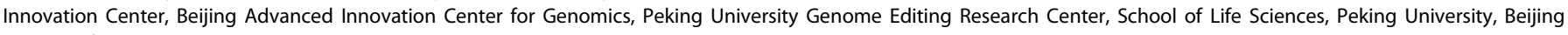
100871, China

Correspondence: Xiaobo Lei (fyleixb@126.com) or Zichun Xiang (xiangzch@163.com) or Jianwei Wang (wangjw28@163.com)

These authors contributed equally: Wenjing Wang, Zhuo Zhou, Xia Xiao.
}

Received: 13 September 2020 Accepted: 8 December 2020

Published online: 26 February 2021 
Here, we show that SARS-CoV-2 nsp12 attenuates type I IFN responses by inhibiting IRF3 nuclear translocation. To our knowledge, this is the first demonstration that coronavirus RdRp can antagonize type I IFN responses in addition to exhibiting its polymerase and nucleotidyltransferase activities.

\section{RESULTS}

SARS-CoV-2 nsp12 attenuates type I IFN activation

To examine whether SARS-CoV-2 nsp12 could regulate innate immune responses, we first evaluated the effect of nsp12 on IFN- $\beta$ promoter activation. 293T cells were transiently transfected with a vector plasmid or plasmids expressing nsp 12 along with an IFN- $\beta$ promoter-driven luciferase reporter plasmid (pIFN$\beta$-Luc) and a control pRL-TK plasmid. After $24 \mathrm{~h}$, the cells were stimulated with $\mathrm{SeV}$ or high-molecular weight poly(l:C) (HMWpoly[l:C]), which activates the RIG-I and MDA5 signaling pathways, respectively. ${ }^{18,24}$ The luciferase activity was determined $12 \mathrm{~h}$ post stimulation. We found that nsp12 inhibited SeV- or HMW-poly(I:C)-induced IFN- $\beta$ promoter activation in a dosedependent manner (Fig. 1a, b). Moreover, this inhibition was observed when the endogenous expression of IFN- $\beta$ was examined by RT-PCR assays, verifying that nsp12 inhibits the RIG-I and MDA5 signaling pathways (Fig. 1C). Overexpression of nsp12 also inhibited SeV-stimulated STAT1 phosphorylation, an event following IFN- $\beta$ production (Fig. 1d). We further investigated whether nsp12 affects signaling pathways downstream of IFN- $\beta$ production. 293T cells were transiently transfected with a vector plasmid or with plasmids expressing nsp 12 along with an interferon-stimulated response element (ISRE) reporter plasmid and a control pRL-TK plasmid. After $24 \mathrm{~h}$, the cells were stimulated with IFN- $\beta$ for $12 \mathrm{~h}$, and the luciferase activity was determined. We found that nsp12 could not inhibit IFN- $\beta$ induced ISRE promoter activation (Fig. 1e), suggesting that nsp12 perturbs IFN- $\beta$ activation rather than downstream signaling.

SARS-CoV-2 nsp12 inhibits IRF3-triggered IFN- $\beta$ activation To identify the host targets on which SARS-CoV-2 nsp12 exerts its inhibitory effect, we cotransfected increasing amounts of nsp12 with RIG-IN (an active form of RIG-I), MDA5, MAVS, and IRF3-5D (a constitutively active IRF3 mutant) and determined the activation of the IFN- $\beta$ promoter. Luciferase reporter assays showed that overexpression of nsp12 inhibited RIG-IN-, MDA5-, MAVS-, and IRF3-5D-triggered IFN- $\beta$ promoter activation in a dose-dependent manner (Fig. 2a-d). These results demonstrated that nsp12 inhibited IFN- $\beta$ production at the level of or downstream of IRF3 activation.

SARS-CoV-2 nsp12 does not inhibit the phosphorylation of IRF3 IRF3 is activated after phosphorylation by the kinases IKKi and TBK1. ${ }^{23}$ To determine whether SARS-CoV-2 nsp12 antagonizes IRF3 phosphorylation, 293T cells were transiently transfected with a vector plasmid or with plasmids expressing nsp12. After $24 \mathrm{~h}$, the cells were stimulated with SeV for different durations (Fig. 3a). Alternatively, cells were transfected with increasing amounts of plasmids expressing nsp 12 for $24 \mathrm{~h}$ and were then infected with SeV for $8 \mathrm{~h}$ (Fig. 3b). Sev induced IRF3 phosphorylation at comparable levels in the presence or absence of nsp12 (Fig. 3a, b) in various settings, suggesting that SARS-CoV-2 nsp12 does not affect virus-triggered IRF3 phosphorylation.

SARS-CoV-2 nsp12 suppresses the nuclear translocation of IRF3 The translocation of phosphorylated IRF3 into the nucleus is critical for IFN- $\beta$ transcription. Since nsp12 does not affect IRF3 phosphorylation, we then examined whether nsp12 affects IRF3 nuclear translocation in 293T cells. Immunofluorescence analyses showed that in uninfected cells, IRF3 was distributed in the cytoplasm with or without overexpression of nsp12 (Fig. 4a, row 1 and 2). In cells transfected with control vectors, IRF3 was efficiently translocated to the nucleus upon SeV infection (Fig. 4a, row 3). However, SeV-induced IRF3 nuclear translocation was severely impaired in cells expressing nsp12 (Fig. 4a (row 4), b). Similar results were observed in HeLa-ACE2 cells that are permissive to SARS-CoV-2 infection (Supplementary Fig. 1). Nuclear fractionation assays further confirmed this observation, showing that nsp12 overexpression substantially suppressed SeV-triggered nuclear accumulation of IRF3 (Fig. 4c). These data support that SARS-CoV-2 nsp12 can inhibit IRF3 nuclear translocation in response to virus infection. Moreover, this nsp12-mediated inhibition was recapitulated when IRF3 nuclear translocation was stimulated by RIG-IN overexpression (Fig. 4d).

We then investigated whether nsp12 exerts its inhibitory effect by physically interacting with IRF3. Coimmunoprecipitation assays showed that nsp12 did not associate with IRF3 in the presence or absence of SeV infection (Supplementary Fig. 2a). Serving as the positive control, TBK1 was efficiently coimmunoprecipitated with IRF3 (Supplementary Fig. 2b).

Nsp12 inhibits IFN- $\beta$ production via a mechanism independent of its enzymatic activity

As nsp12 is responsible for catalyzing the synthesis of viral RNA, we explored whether its enzymatic activity is involved in IFN regulation. First, we investigated whether the RdRp activity of SARS-CoV-2 nsp12 is required for inhibiting the nuclear translocation of IRF3 upon SeV infection. Two nsp12 variants harboring mutations in the putative $\mathrm{RdRp}$ catalytic residues found in coronavirus and other RNA viruses, including SDD-AAA (in which amino acids 759-761 are substituted with alanine residues) and G823V (in which amino acid 823 is substituted with valine), were generated. The effects of wild-type nsp12 and nsp12 mutants on IFN promoter activation and the nuclear translocation of IRF3 were then assayed. We found that the SDD-AAA and G823V mutants of nsp12 suppressed SeV-induced IFN- $\beta$ promoter activation to a level comparable to that of wild-type nsp12 (Fig. 5a). These two mutants also suppressed the nuclear translocation of IRF3 upon SeV infection (Fig. 5b, c).

To further examine whether polymerase activity is associated with nsp12's antagonistic function, we tested the effect of remdesivir, a nucleoside analog antiviral drug that inhibits the polymerase activity of nsp $12 .^{7}$ Remdesivir efficiently inhibited SARS-CoV-2 replication in cell culture (Fig. $5 \mathrm{~d}$ left panel); however, it failed to block nsp12's antagonistic activity on IFN- $\beta$ activation triggered by the overexpression of IRF3-5D (Fig. 5d right panel). On the basis of the collective data, we conclude that SARS-CoV-2 nsp12 can suppress IFN- $\beta$ activation in a polymerase activityindependent manner.

We then examined the effect of the nsp12 NiRAN domain, which is proposed to have nucleotidyltransferase activity. To do this, we constructed a nsp12 mutant devoid of the NiRAN domain ( $\triangle$ NiRAN). 293T cells were transiently transfected with a vector plasmid or plasmids expressing nsp12 or $\triangle$ NiRAN along with pIFN$\beta$-Luc and pRL-TK. After $24 \mathrm{~h}$, the cells were stimulated with SeV for $12 \mathrm{~h}$. We found that the $\triangle$ NiRAN mutant exerted an inhibitory effect on IFN comparable to that of wild-type nsp12 (Fig. 6), suggesting that the NiRAN domain is not involved in IFN suppression.

The coronavirus polymerase complex is composed of nsp 12 and two cofactors, nsp7 and nsp8. ${ }^{26}$ We next texted whether these two cofactors play roles in nsp12-mediated IFN suppression. We found that although $\mathrm{nsp} 7$ and $\mathrm{nsp} 8$ are reported to promote the polymerase activity of $n s p 12,{ }^{27}$ they failed to potentiate nsp12's inhibitory activity on IFN (Supplementary Fig. 3), further suggesting that nsp12 functions as an IFN antagonist independently. 
a
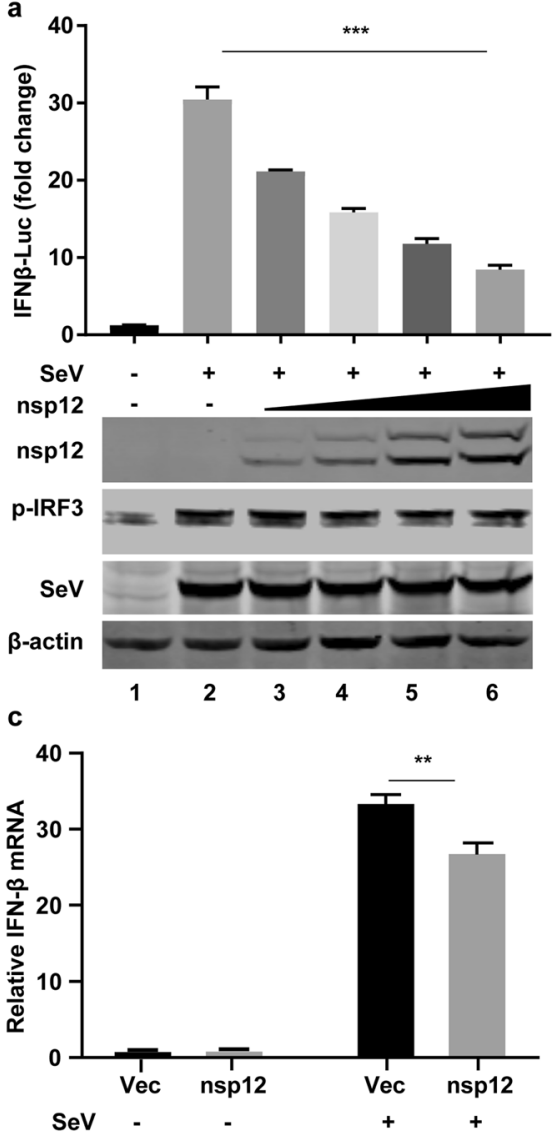

d

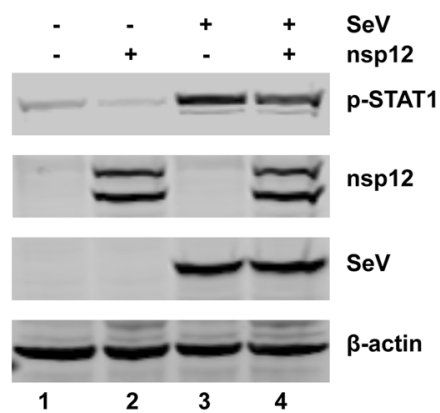

b
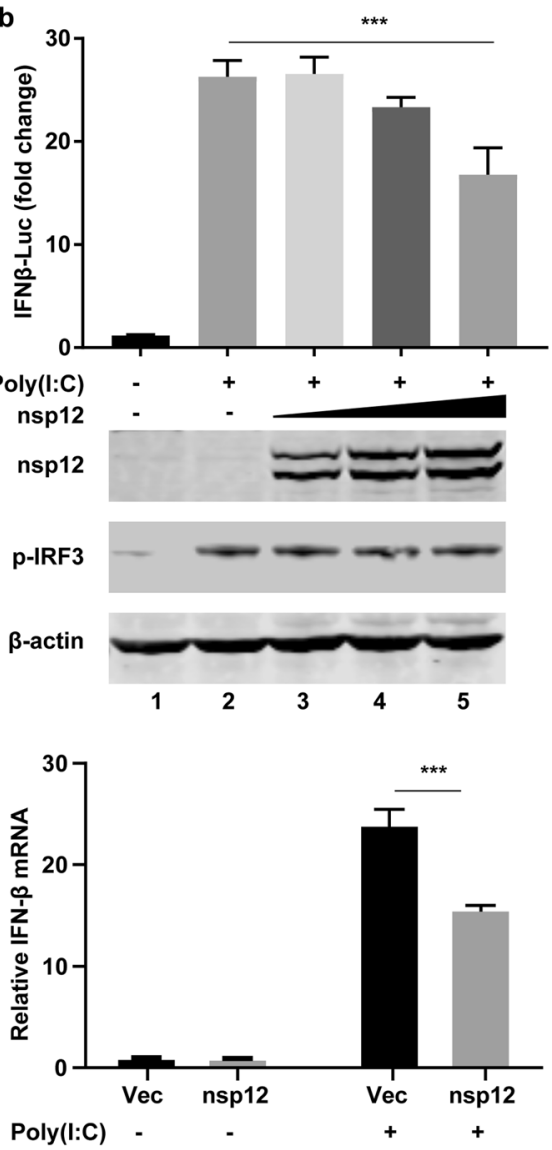

e

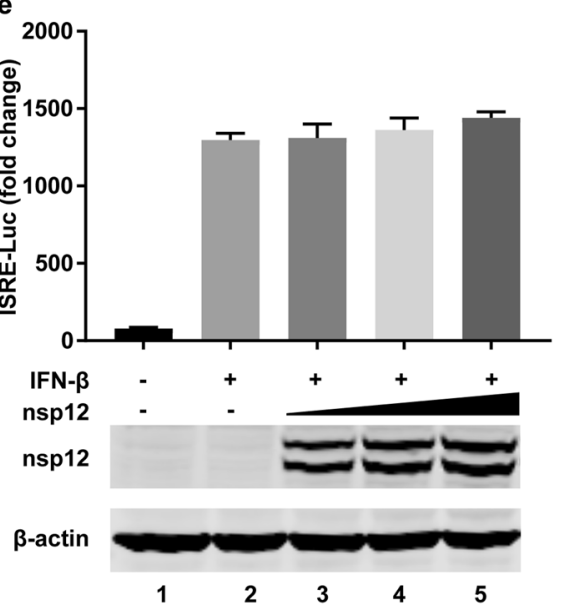

Fig. 1 SARS-CoV-2 nsp12 attenuates viral RNA-related type I interferon responses. a Effects of nsp12 on SeV-induced IFN- $\beta$ promoter activation. HEK293T cells were transfected with an IFN- $\beta$ reporter plasmid along with a control plasmid or with increasing amounts of plasmids expressing $\mathrm{nsp12}$. The cells were infected with SeV for $12 \mathrm{~h}$ and assayed for luciferase activity. The indicated protein expression levels were analyzed by western blotting. p-IRF3 was used to assess the stimulation after SeV infection. b Effects of nsp 12 on high-molecular weight poly(l:C)-induced IFN- $\beta$ promoter activation. HEK293T cells were transfected with an IFN- $\beta$ reporter plasmid along with a control plasmid or with increasing amounts of plasmids expressing nsp12. The cells were transfected with high-molecular weight poly(l:C) for $12 \mathrm{~h}$ and assayed for luciferase activity. The indicated protein expression levels were analyzed by western blotting. p-IRF3 was used to assess the stimulation of poly I:C treatment. c Effect of nsp12 on endogenous IFN- $\beta$ mRNA expression induced by SeV (left) and high-molecular weight poly(l:C) (right). HEK293T cells were transfected with a control plasmid or a plasmid expressing nsp12. After $24 \mathrm{~h}$, cells were infected with SeV for $8 \mathrm{~h}$ or transfected with high-molecular weight poly(l:C) for $6 \mathrm{~h}$. Total RNA was extracted, and the expression of IFN- $\beta$ was detected by real-time RT-PCR. d Effect of nsp12 on endogenous p-STAT1 induced by SeV. HEK293T cells were transfected with a control plasmid or a plasmid expressing nsp12. After $24 \mathrm{~h}$, the cells were infected with SeV for $8 \mathrm{~h}$. Whole-cell lysates were analyzed by western blotting for p-STAT1, nsp12, and P protein of SeV. e Effects of nsp12 on IFN-induced ISRE promoter activation. HEK293T cells were transfected with an ISRE-Luc reporter plasmid along with a control plasmid or with increasing amounts of plasmids expressing nsp12. After $24 \mathrm{~h}$, the cells were treated with IFN $(1000 \mathrm{U} / \mathrm{ml})$ for $12 \mathrm{~h}$ and assayed for luciferase activity. The indicated protein expression levels were analyzed by western blotting. All experiments were performed at least twice, and one representative is shown. The error bars indicate the SDs of technical triplicates. One-way ANOVA (or the nonparametric equivalent) was used for column analyses (a, $\mathbf{b}$ ). The two-tailed unpaired $t$-test was used for two-group comparisons (c). ${ }^{* *} P<0.01,{ }^{* * *} P<0.001$ 

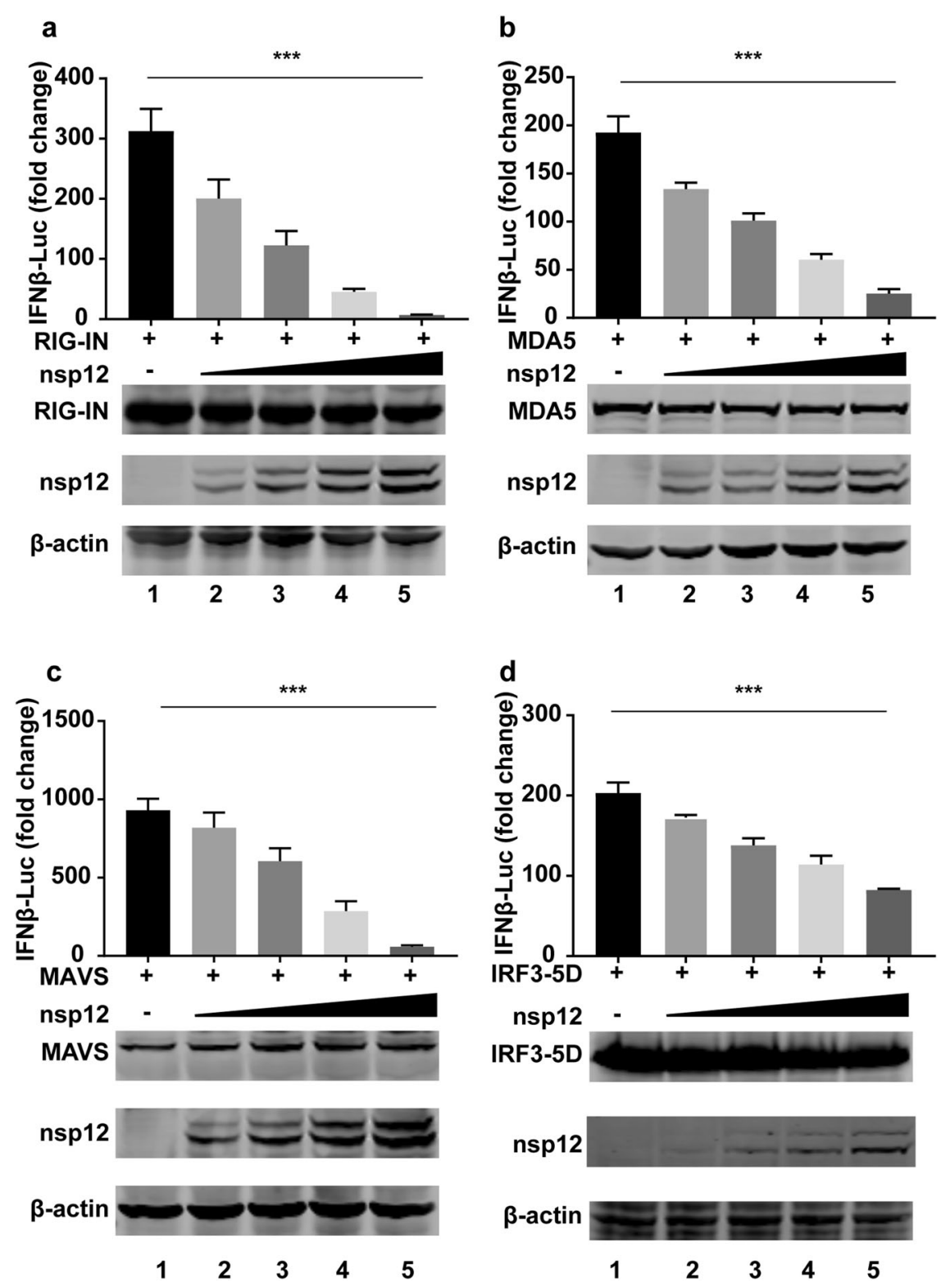

Fig. 2 SARS-CoV-2 nsp12 inhibits IRF3 activation. Effects of nsp12 on RIG-IN-, MDA5-, MAVS-, or IRF3-induced IFN- $\beta$ promoter activation. HEK293T cells were transfected with an IFN- $\beta$ reporter plasmid along with a control plasmid or with increasing amounts of plasmids expressing nsp12 together with plasmids expressing RIG-IN (a), MDA5 (b), MAVS (c), or IRF3-5D (d). At 24 h post transfection, luciferase activity was measured. The expression levels of the indicated proteins were analyzed by western blotting. All experiments were performed at least twice, and one representative is shown. The error bars indicate the SDs of technical triplicates. One-way ANOVA (and the nonparametric equivalent) was used for column analyses, ${ }^{* * *} P<0.001$

\section{DISCUSSION}

SARS-CoV-2 infection induces the production of aberrant type I IFN in cultured cells and COVID-19 patients. ${ }^{28}$ This phenomenon is at least partly due to the various viral mechanisms for evasion and suppression of IFN activation. ${ }^{29,30}$ Several SARS-CoV-2 proteins, including nsp1, nsp3, nsp6, nsp8, nsp12, nsp13, nsp14, nsp15, ORF3, ORF6, ORF8, ORF9b, M, and N, have been reported to inhibit type I IFN activation and the corresponding response. $^{14,31-39}$ Here, we report that nsp12 of SARS-CoV-2 attenuates type I IFN induction by inhibiting IRF3 nuclear translocation.

In our and other groups' efforts to screen viral proteins that potentially regulate type I IFN, ${ }^{14,38}$ multiple nsps, including nsp12, nsp13, nsp14, and nsp15, have been found to exert antagonistic activities. These nsps are immediately translated from incoming viral genomic RNAs. We postulate that these "early" viral proteins, including nsp12, may help to antagonize the host innate immune response triggered by incoming viral RNAs or other signals at the beginning of infection. These proteins are then recruited to double-membrane vesicles, where they form a replication/transcription complex to catalyze viral genomic replication and transcription. Then, "late" viral proteins are translated from subgenomic RNAs, including ORF3, ORF6, and $M$, which exert potent inhibitory effects on host IFN activation and signaling.

The polymerases of other RNA viruses have been reported to regulate type I IFN activation. 3D pol of enterovirus 71 and coxsackievirus B3 interact with the caspase activation and 
a

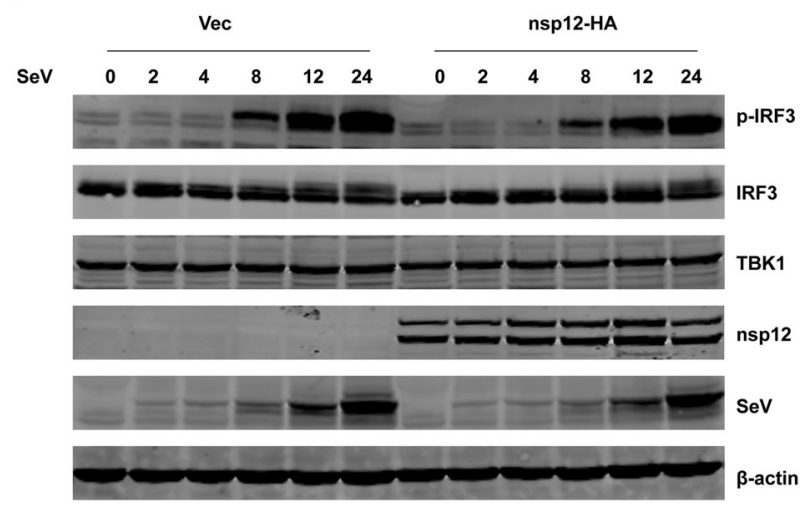

b

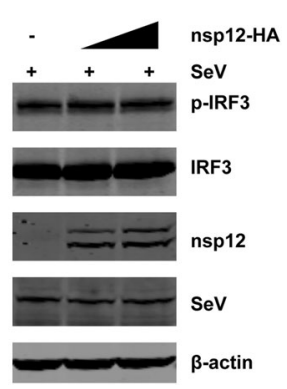

Fig. 3 SARS-CoV-2 nsp12 does not inhibit the phosphorylation of IRF3. HEK293T cells were transfected with a control plasmid or nsp12 expression plasmid. After $24 \mathrm{~h}$, the cells were infected with $\mathrm{SeV}$ and collected at the indicated times. Whole-cell lysates were analyzed by western blotting for p-IRF3, IRF3, TBK1, nsp12, and $P$ protein of SeV. b HEK293T cells were transfected with a control plasmid or increasing amounts of plasmids expressing nsp12. After $24 \mathrm{~h}$, the cells were infected with SeV for $8 \mathrm{~h}$. Whole-cell lysates were analyzed by western blotting for p-IRF3, IRF3, nsp12, and $\mathrm{P}$ protein of $\mathrm{SeV}$

recruitment domains of MDA5 to inhibit IFN- $\beta$ promoter activation and mRNA expression. ${ }^{10}$ Similar to the case for SARS-CoV-2 nsp12, the polymerase activity of EV71 $3 D^{\text {pol }}$ is not required to suppress type I IFN activation. ${ }^{10}$ Polymerases of flaviviruses such as hepatitis $C$ virus, tick-borne encephalitis virus, and Langat virus regulate the type I IFN response via RdRp activity. ${ }^{11-13}$ These findings indicate that viral polymerase could play pleiotropic roles in addition to exerting RdRp activity. It is unclear how nsp12 inhibits IRF3 nuclear translocation, and the underlying mechanism awaits further investigation.

In summary, we demonstrate that SARS-CoV-2 nsp12 attenuates type I IFN activation by inhibiting IRF3 nuclear translocation. This inhibitory effect is independent of nsp12 polymerase activity. Further understanding of the role and mechanism of action of nsp12 may direct us to novel therapeutic targets.

\section{METHODS}

Cell lines and viruses

Human 293T (ATCC, \#CCL-11268), HeLa (ATCC, \#CCL-2), and Vero (ATCC, \#CCL-81) cells were cultured in Dulbecco's modified Eagle's medium (Invitrogen, Carlsbad, CA) supplemented with 10\% heatinactivated fetal bovine serum (FBS) (HyClone, Logan, UT), $100 \mathrm{U} / \mathrm{ml}$ penicillin, and $100 \mathrm{U} / \mathrm{ml}$ streptomycin at $37^{\circ} \mathrm{C}$ in a $5 \% \mathrm{CO}_{2}$ humidified atmosphere. Low-passage cells were used after direct purchase from ATCC, and all cells were mycoplasma-free. SARS-CoV-2 was isolated from respiratory samples of confirmed COVID-19 patients by inoculation onto Vero cells ${ }^{1}$ and was propagated in Vero cells for use in this study. Cells were infected with SARS-CoV-2 at a multiplicity of infection (MOl) of 0.1 or 0.5 . Unbound virus was washed away after $1 \mathrm{~h}$, and cells were then cultured with fresh medium supplemented with $2 \%$ FBS. All experiments with SARS-CoV-2 were conducted in the BSL-3 laboratory.

Plasmids and antibodies

The SARS-CoV-2 nsp12 gene (IPBCAMS-WH-01/2019 strain, no. EPI_ISL_402123) was optimized by Gene Designer 1.0 and cloned into the pCMV6-Entry expression vector with an HA-tag at the $\mathrm{C}$ terminus. The plasmids Flag-RIG-IN, Flag-MDA5, HA-MAVS, pGL3IFN- $\beta$-Luc, IRF3-5D-Flag, and pRL-TK have been described elsewhere. ${ }^{40}$ Mutated variants of SARS-CoV-2 nsp12-tagged HA were constructed by using a site-directed mutagenesis kit (Stratagene, La Jolla, CA). All variants were confirmed by subsequent sequencing.

The antibodies used in this research included the following: a SARS-CoV-2 (COVID-19) RdRp (nsp12) antibody from GeneTex (1:1000, Cat\#GTX135467), a Flag antibody from SigmaAldrich (1:4000, Cat\# F3165), a $\beta$-actin antibody from SigmaAldrich (1:4000, Cat\# A5441), an HA antibody from Sigma-Aldrich (1:10000, Cat\# H9658); a p-STAT1 antibody from Thermo Fisher (1:1000, Cat\# 700349), a TBK1 antibody from Cell Signaling Technology (1:1000, Cat\# 3504), an IRF3 antibody from Abcam (1:1000, Cat\# 76409) a p-IRF3 antibody (S386) from Abcam (1:1000, Cat\# 76493), a Lamin A antibody from SigmaAldrich (1:2000, Cat\# L1293), a $\beta$-tubulin antibody from Zsbio (1:1000, Cat\# TA-10), and a SeV antibody from MBL (1:2000, PD029(1). A Dual-Luciferase Reporter Assay System was purchased from Promega (Madison, WI). IRDye 800 -labeled IgG and IRDye 680-labeled IgG secondary antibodies were purchased from LI-COR Biosciences (Lincoln, NE).

Reporter assays

293T cells cultured in 24-well plates were transfected with luciferase reporter plasmids or plasmids expressing indicated proteins. Cells were harvested, and the cell lysates were used to determine luciferase activity using a Dual Luciferase Reporter Assay System (Promega). The firefly luciferase activity levels were normalized to the Renilla luciferase activity levels. ${ }^{40}$

Western blot analysis

The samples were separated on denaturing SDS-PAGE gels and transferred electrophoretically onto nitrocellulose membranes. Proteins of interest were immunoblotted with the indicated primary antibodies and IRDye secondary antibodies (LI-COR Biosciences, Lincoln, NE). The protein expression levels were detected by using the Odyssey Infrared Imaging System (LI-COR Biosciences) and analyzed by using the integrated software of the Odyssey system (Image Studio Ver 5.2).

Immunofluorescence

Cells were washed with PBS and fixed with $4 \%$ paraformaldehyde. Then, the cells were permeabilized with $0.5 \%$ Triton X-100. After the cells were washed with PBS, they were blocked and stained with primary antibodies before being stained with Alexa Fluor 488-, 594- and 647-conjugated secondary antibodies. ${ }^{41}$ Nuclei were stained with DAPI (Sigma). The antibodies used in this research were an IRF3 antibody from Cell Signaling Technology (1:200, Cat\# 11904), an HA antibody from SigmaAldrich (1:100, Cat\# H9658), and a Myc antibody from SigmaAldrich (1:200, Cat\# M4439). Fluorescence images were obtained and analyzed using a laser scanning confocal microscope (Zeiss LSM 800).

Quantitative real-time PCR analysis

Total RNA was extracted by using TRIzol reagent (Invitrogen, Carlsbad, CA) and reverse transcribed into CDNA by M-MLV Reverse Transcriptase (Promega, Madison, WI). The cDNA was 


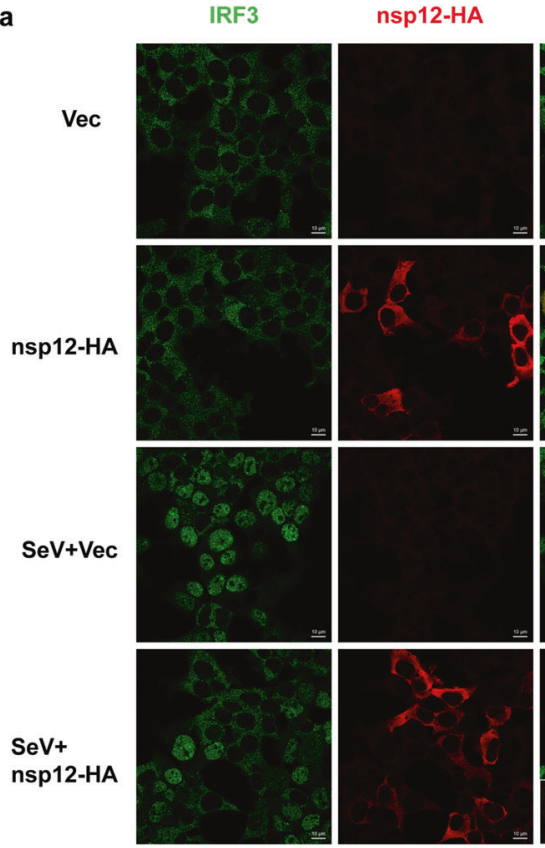

b

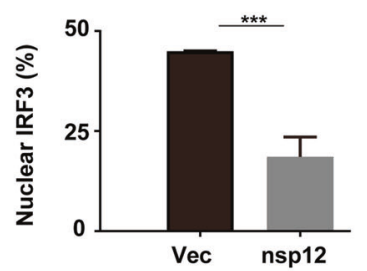

d

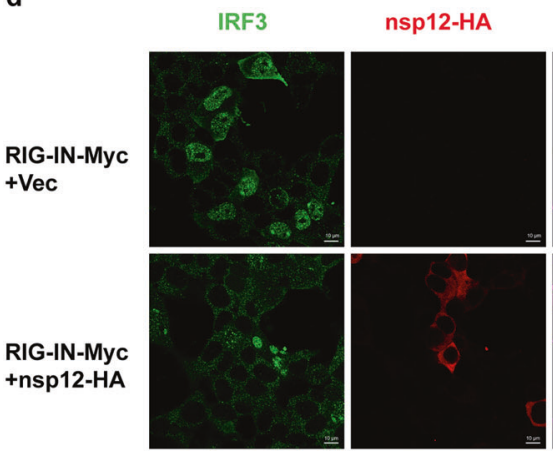

Merge1
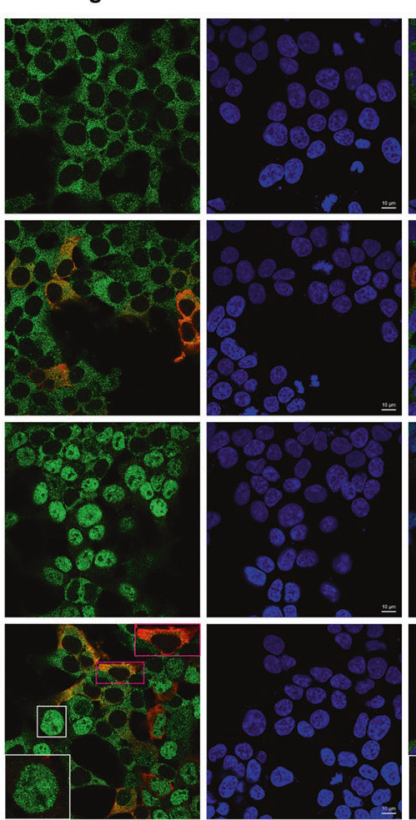

C

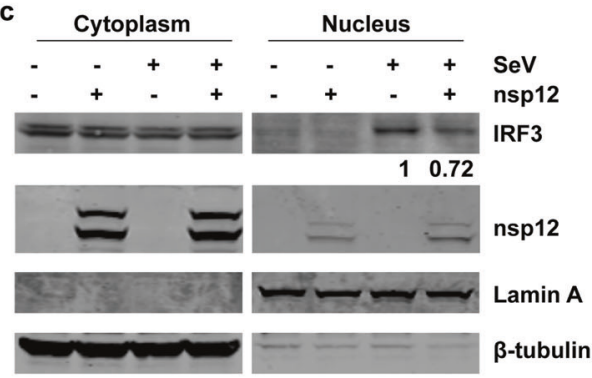

RIG-IN-Myc
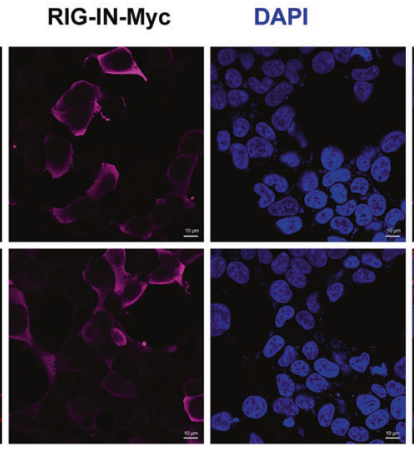

Merge

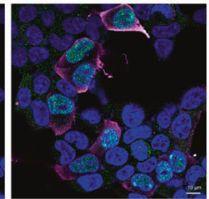

Merge2
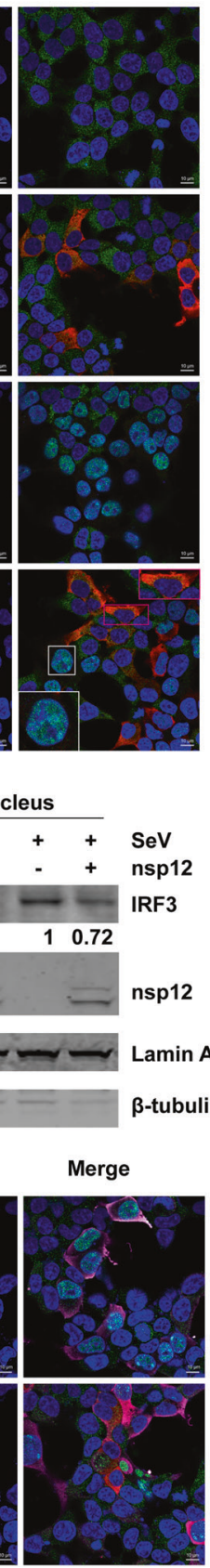

Fig. 4 SARS-CoV-2 nsp12 suppresses the nuclear translocation of IRF3. a Effect of SARS-CoV-2 nsp12 on SeV-induced nuclear translocation of IRF3. HEK293T cells were transfected with a control plasmid or an nsp12 expression plasmid. At $24 \mathrm{~h}$ post transfection, the cells were infected with $\mathrm{SeV}$ for $4 \mathrm{~h}$ and then immunostained with the indicated antibodies. Merge 1 and Merge 2 indicate the merged red and green channels and the merged red, green, and blue channels, respectively. Scale bar, $10 \mu \mathrm{M}$. The enlarged image is a magnified view of the corresponding box. b Quantitation of the nuclear translocation of IRF3. The percentage of nuclear IRF3-positive transfected cells was determined in three independent experiments. The two-tailed unpaired $t$-test was used for two-group comparisons, $* * *<0.001$. c Immunoblot analysis of cytoplasmic and nuclear IRF3 after SeV infection. HEK293T cells were transfected with a control plasmid or an nsp12 expression plasmid. After $24 \mathrm{~h}$, the cells were infected with SeV for $4 \mathrm{~h}$ and fractionated into cytoplasmic and nuclear fractions. The fractions were analyzed by western blotting for IRF3 and nsp12 detection. $\beta$-Tubulin and Lamin A were used as a cytoplasmic and a nuclear marker, respectively. The numbers under the lanes indicate the IRF3 band intensity, which was normalized to that of Lamin A. d Effect of SARS-CoV-2 nsp12 on RIG-IN-induced nuclear translocation of IRF3. HEK293T cells were transfected with RIG-IN or RIG-IN and nsp12 expression plasmids. At $24 \mathrm{~h}$ post transfection, the cells were immunostained with the indicated antibodies

prepared for real-time PCR by using TB Green Premix Ex (Takara, Kusatsu, Shiga). The primer sequences used for IFN $\beta$ are $5^{\prime}$ TAGCACTGGCTGGAATGAG3' (forward) and 5'GTITCGGAGGTAA CCTGTAAG $3^{\prime}$ (reverse). ${ }^{41}$

\section{Statistics}

One-way ANOVA or the nonparametric equivalent was used for column analyses. The two-tailed unpaired $t$-test was used for twogroup comparisons. ${ }^{* *} P<0.01$, ${ }^{* *} P<0.001$. 
a

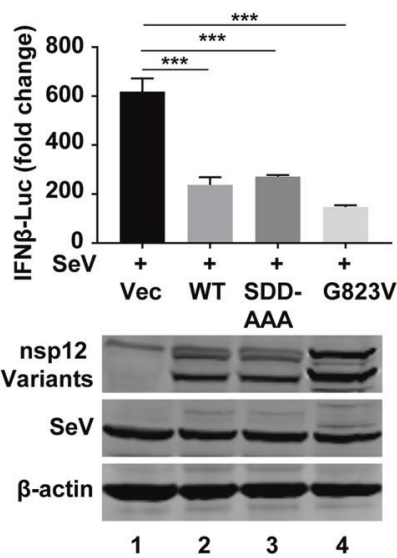

C

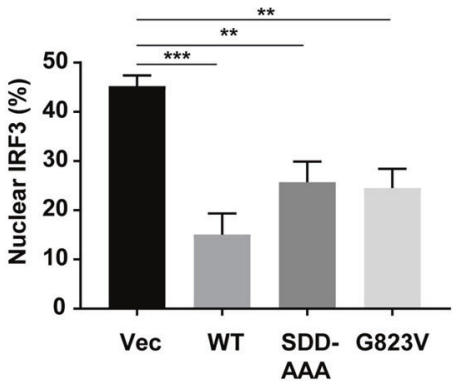

DAPI
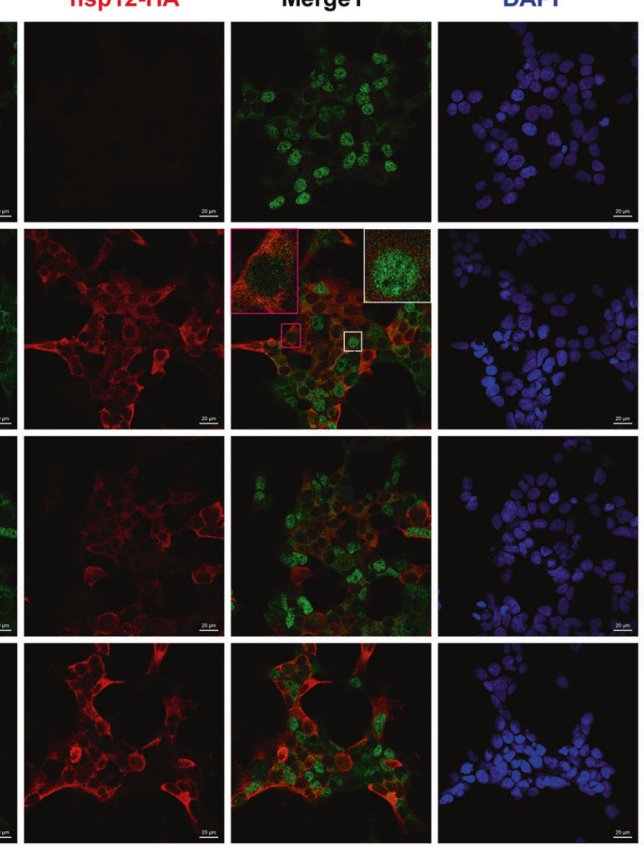

d
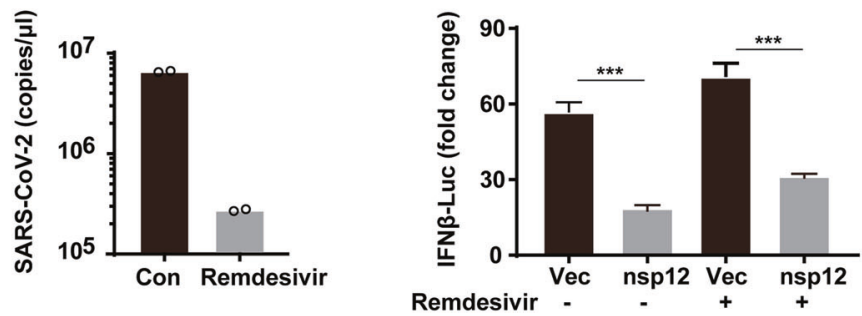

Fig. 5 The polymerase activity of SARS-CoV-2 nsp12 is not required for suppression of type I IFN activation. a Effects of SARS-CoV-2 nsp12 and its mutants on SeV-induced IFN- $\beta$ promoter activation. HEK293T cells were transfected with an IFN- $\beta$ reporter plasmid along with a control plasmid or plasmids expressing wild-type SARS-CoV-2 nsp12 or the indicated SARS-CoV-2 nsp12 variants. The cells were infected with SeV for $12 \mathrm{~h}$ and assayed for luciferase activity. The expression levels of the indicated proteins were analyzed by western blotting. $\mathbf{b}$ Effect of SARSCoV-2 nsp12 and its mutants on SeV-induced nuclear translocation of IRF3. HEK293T cells were transfected with a control plasmid or plasmids expressing wild-type SARS-CoV-2 nsp12 or the indicated SARS-CoV-2 nsp12 variants. At $24 \mathrm{~h}$ post transfection, the cells were infected with SeV for $4 \mathrm{~h}$ and then immunostained with the indicated antibodies. Merge 1 and Merge 2 indicate the merged red and green channels and the merged red, green, and blue channels, respectively. Scale bar, $20 \mu \mathrm{M}$. The enlarged image is a magnified view of the corresponding box. c Quantitation of the nuclear translocation of IRF3. d Vero cells were infected with SARS-CoV-2 at an MOI of 0.1 with or without $10 \mu \mathrm{M}$ remdesivir for $24 \mathrm{~h}$. The viral copies in the supernatants were measured using real-time RT-PCR (left panel). HEK293T cells were transfected with an IFN- $\beta$ reporter plasmid along with a control plasmid or nsp12 expression plasmid and a plasmid expressing IRF3-5D. Remdesivir $(10 \mu \mathrm{M})$ was added to the culture medium at $5 \mathrm{~h}$ post transfection. At $24 \mathrm{~h}$ post transfection, luciferase activity was measured (right panel). All experiments were performed at least twice, and one representative is shown. The error bars indicate the SDs of technical triplicates. The twotailed unpaired $t$-test was used for two-group comparisons compared to the vector group, ${ }^{* *} P<0.01,{ }^{* * *} P<0.001$ 
a

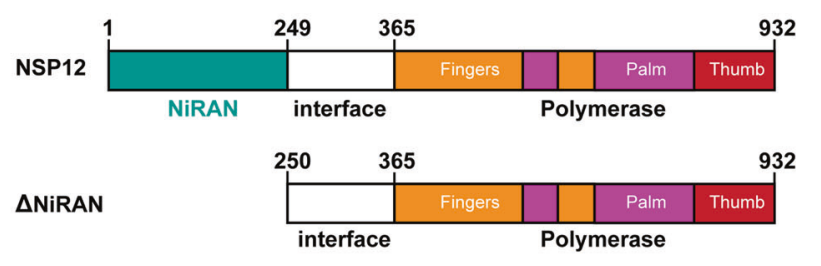

b

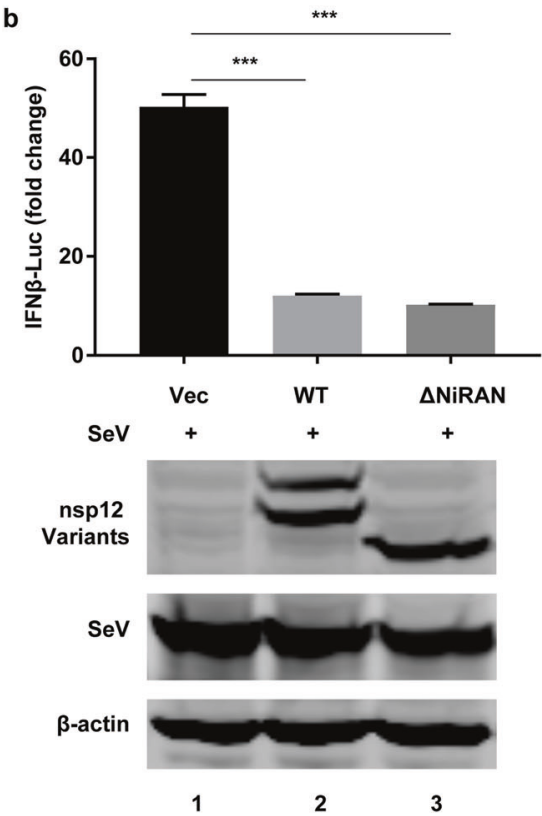

Fig. 6 IFN regulation by SARS-CoV-2 nsp12 is not related to the NiRAN domain. a Schematic diagram of SARS-CoV-2 nsp12. b Effects of SARS-CoV-2 nsp12 and the $\Delta$ NiRAN mutant on SeV-induced IFN- $\beta$ promoter activation. HEK293T cells were transfected with an IFN- $\beta$ reporter plasmid along with a control plasmid or plasmids expressing wild-type nsp12 or the $\Delta$ NiRAN mutant. The cells were infected with SeV for $12 \mathrm{~h}$ and assayed for luciferase activity. The expression levels of the indicated protein were analyzed by western blotting. The two-tailed unpaired t-test was used for two-group comparisons compared to the vector group, ${ }^{* *} P<0.001$

\section{DATA AVAILABILITY}

All data related to this paper may be requested from the authors.

\section{ACKNOWLEDGEMENTS}

This work was supported by grants from the National Major Sciences \& Technology Project for Control and Prevention of Major Infectious Diseases in China (2018ZX10733403 and 2018ZX10101001 to Z.X., 2018ZX10301401 to X.L. and Z.Z.), the National Natural Science Foundation of China (81930063, 81971948, 81772201, and 31670169 to J.W., X.L., Z.X., and Z.Z.), the National Key R\&D Program of China (2020YFA0707600), and the Chinese Academy of Medical Sciences (CAMS) Innovation Fund for Medical Sciences (2016-12M-1-014 to J.W.).

\section{AUTHOR CONTRIBUTIONS}

Project conception: Z.X., X.L., and J.W.; experimental design: Z.X., X.L., L.R., Z.Z, and J.W.; experimental work: W.W., X.X., Z.T., C.W., X.D., and L.L.; data analysis: Z.X., X.L., L.R., Z.Z., and J.W., writing of original draft: Z.X., W.W., X.L., Z.Z., and J.W.; writing, review \& editing: J.W., X.L., Z.Z., and Z.X. All authors reviewed the manuscript.

\section{ADDITIONAL INFORMATION}

The online version of this article (https://doi.org/10.1038/s41423-020-00619-y) contains supplementary material.

Competing interests: The authors declare no competing interests.

\section{REFERENCES}

1. Ren, L. L. et al. Identification of a novel coronavirus causing severe pneumonia in human: a descriptive study. Chin. Med. J. 133, 1015-1024 (2020).

2. Zhou, P. et al. A pneumonia outbreak associated with a new coronavirus of probable bat origin. Nature 579, 270-273 (2020).

3. Zhu, N. et al. A novel coronavirus from patients with pneumonia in China, 2019. N. Engl. J. Med. 382, 727-733 (2020).

4. Coronaviridae Study Group of the International Committee on Taxonomy of Viruses. The species severe acute respiratory syndromerelated coronavirus: classifying 2019-nCoV and naming it SARS-CoV-2. Nat. Microbiol. 5, 536-544 (2020).

5. de Wilde, A. H., Snijder, E. J., Kikkert, M. \& van Hemert, M. J. Host factors in coronavirus replication. Curr. Top Microbiol. Immunol. 419, 1-42 (2018).

6. Knoops, K. et al. SARS-coronavirus replication is supported by a reticulovesicular network of modified endoplasmic reticulum. PLoS Biol. 6(9), e226 (2008).

7. Gao, Y. et al. Structure of the RNA-dependent RNA polymerase from COVID-19 virus. Science 368, 779-782 (2020).

8. Appleby, T. C. et al. Structural basis for RNA replication by the hepatitis $C$ virus polymerase. Science 347, 771-775 (2015).

9. Gong, P. \& Peersen, O. B. Structural basis for active site closure by the poliovirus RNA-dependent RNA polymerase. Proc. Natl Acad. Sci. USA 107, 22505-22510 (2010).

10. Kuo, R. L. et al. Role of enteroviral RNA-dependent RNA polymerase in regulation of MDA5-mediated beta interferon activation. J. Virol. 93, e00132-19 (2019).

11. Moriyama, M. et al. Interferon-beta is activated by hepatitis $C$ virus NS5B and inhibited by NS4A, NS4B, and NS5A. Hepatol. Int. 1, 302-310 (2007).

12. Zheng, Z. et al. Tick-borne encephalitis virus nonstructural protein NS5 induces RANTES expression dependent on the RNA-dependent RNA polymerase activity. J. Immunol. 201, 53-68 (2018).

13. Harmon, B. et al. Identification of residues critical for the interferon antagonist function of Langat virus NS5 reveals a role for the RNA-dependent RNA polymerase domain. J. Biol. Chem. 288, 8702-8711 (2013).

14. Lei, X. et al. Activation and evasion of type I interferon responses by SARS-CoV-2. Nat. Commun. 11, 3810 (2020).

15. Akira, S., Uematsu, S. \& Takeuchi, O. Pathogen recognition and innate immunity. Cell 124, 783-801 (2006).

16. Alexopoulou, L., Holt, A. C., Medzhitov, R. \& Flavell, R. A. Recognition of doublestranded RNA and activation of NF-kappaB by Toll-like receptor 3. Nature 413, 732-738 (2001).

17. Medzhitov, R., Preston-Hurlburt, P. \& Janeway, C. A. Jr. A human homologue of the Drosophila Toll protein signals activation of adaptive immunity. Nature $\mathbf{3 8 8}$, 394-397 (1997).

18. Pichlmair, A. et al. RIG-I-mediated antiviral responses to single-stranded RNA bearing 5'-phosphates. Science 314, 997-1001 (2006).

19. Kawai, T. et al. IPS-1, an adaptor triggering RIG-I- and Mda5-mediated type I interferon induction. Nat. Immunol. 6, 981-988 (2005).

20. Meylan, E. et al. Cardif is an adaptor protein in the RIG-I antiviral pathway and is targeted by hepatitis C virus. Nature 437, 1167-1172 (2005).

21. Seth, R. B., Sun, L., Ea, C. K. \& Chen, Z. J. Identification and characterization of MAVS, a mitochondrial antiviral signaling protein that activates NF-kappaB and IRF 3. Cell 122, 669-682 (2005).

22. $\mathrm{Xu}, \mathrm{L}$. G. et al. VISA is an adapter protein required for virus-triggered IFN-beta signaling. Mol. Cell 19, 727-740 (2005).

23. Fitzgerald, K. A. et al. IKKepsilon and TBK1 are essential components of the IRF3 signaling pathway. Nat. Immunol. 4, 491-496 (2003).

24. Rehwinkel, J. \& Gack, M. U. RIG-l-like receptors: their regulation and roles in RNA sensing. Nat. Rev. Immunol. 13, 1-15 (2020).

25. Yan, N. \& Chen, Z. J. Intrinsic antiviral immunity. Nat. Immunol. 13, 214-222 (2012).

26. Wang, Q. et al. Structural basis for RNA replication by the SARSCoV-2 polymerase. Cell 182, 417-428.e13 (2020).

27. Subissi, L. et al. One severe acute respiratory syndrome coronavirus protein complex integrates processive RNA polymerase and exonuclease activities. Proc. Natl Acad. Sci. USA 111, E3900-E3909 (2014).

28. lanco-Melo, D. et al. Imbalanced host response to SARS-CoV-2 drives development of COVID-19. Cell 181, 1036-1045 (2020)

29. Sa Ribero, M., Jouvenet, N., Dreux, M. \& Nisole, S. Interplay between SARS-CoV-2 and the type I interferon response. PLoS Pathog. 16, e1008737 (2020).

30. Park, A. \& Iwasaki, A. Type I and Type III interferons-induction, signaling, evasion, and application to combat COVID-19. Cell Host Microbe 27, 870-878 (2020).

31. Thoms, M. et al. Structural basis for translational shutdown and immune evasion by the Nsp1 protein of SARS-CoV-2. Science 369, 1249-1255 (2020).

32. Li, J. Y. et al. The ORF6, ORF8 and nucleocapsid proteins of SARS-CoV-2 inhibit type I interferon signaling pathway. Virus Res. 286, 198074 (2020). 
SARS-CoV-2 nsp12 attenuates type I interferon production by inhibiting... W Wang et al.

33. Shin, D. et al. Papain-like protease regulates SARS-CoV-2 viral spread and innate immunity. Nature. 587, 657-662 (2020).

34. Yang, Z. et al. Suppression of MDA5-mediated antiviral immune responses by NSP8 of SARS-CoV-2. Preprint at https://doi.org/10.1101/2020.08.12.247767.

35. Jiang, H. W. et al. SARS-CoV-2 Orf9b suppresses type I interferon responses by targeting TOM70. Cell. Mol. Immunol. 17, 998-1000 (2020).

36. Han, L. et al. SARS-CoV-2 ORF9b Antagonizes Type I and III interferons by targeting multiple components of RIG-I/MDA-5-MAVS, TLR3-TRIF, and CGAS-STING signaling pathways. Preprint at https://doi.org/10.1101/2020.08.16.252973.

37. Xia, H. et al. Evasion of type I interferon by SARS-CoV-2. Cell Rep. 33, 108234 (2020).

38. Yuen, C. K. et al. SARS-CoV-2 nsp13, nsp14, nsp15 and orf6 function as potent interferon antagonists. Emerg. Microbes. Infect. 9, 1418-1428 (2020).

39. Fu, Y. et al. SARS-CoV-2 membrane glycoprotein $M$ antagonizes the MAVSmediated innate antiviral response. Cell. Mol. Immunol. https://doi.org/10.1038/ s41423-020-00571-x. (2020).

40. Lei, $X$. et al. The $3 C$ protein of enterovirus 71 inhibits retinoid acid-inducible gene I-mediated interferon regulatory factor 3 activation and type I interferon responses. J. Virol. 84, 8051-8061 (2010).
41. Xiao, $X$. et al. Enterovirus $3 A$ facilitates viral replication by promoting phosphatidylinositol 4-kinase IIlbeta-ACBD3 interaction. J. Virol. 91, e00791-17 (2017)

(i) Open Access This article is licensed under a Creative Commons Attribution 4.0 International License, which permits use, sharing, adaptation, distribution and reproduction in any medium or format, as long as you give appropriate credit to the original author(s) and the source, provide a link to the Creative Commons license, and indicate if changes were made. The images or other third party material in this article are included in the article's Creative Commons license, unless indicated otherwise in a credit line to the material. If material is not included in the article's Creative Commons license and your intended use is not permitted by statutory regulation or exceeds the permitted use, you will need to obtain permission directly from the copyright holder. To view a copy of this license, visit http://creativecommons. org/licenses/by/4.0/.

(c) The Author(s) 2021 